\title{
Operationalization of bi-directional screening for tuberculosis and diabetes in private sector healthcare clinics in Karachi, Pakistan
}

Mashal S. Basir ${ }^{1 *}$ (D), Shifa S. Habib² , Syed M.A. Zaidi ${ }^{2}$ Saira Khowaja ${ }^{1}$, Hamidah Hussain ${ }^{1}$, Rashida A. Ferrand ${ }^{3}$ and Aamir J. Khan ${ }^{1}$

\begin{abstract}
Background: Many countries are facing overlapping epidemics of tuberculosis (TB) and diabetes mellitus (DM). Diabetes increases the overall risk of developing Tuberculosis (TB) and contributes to adverse treatment outcomes. Active screening for both diseases can reduce TB transmission and prevent the development of complications of DM. We investigated bi-directional TB-DM screening in Karachi, Pakistan, a country that ranks fifth among high TB burden countries, and has the seventh highest country burden for DM.

Methods: Between February to November 2014, community-based screeners identified presumptive TB and DM through verbal screening at private health clinics. Individuals with presumptive TB were referred for a chest X-ray and Xpert MTB/RIF. Presumptive DM cases had random blood glucose (RBS) tested. All individuals with bacteriologically positive TB were referred for diabetes testing (RBS). All pre-diabetics and diabetics were referred for a chest $X$-ray and Xpert MTB/RIF test. The primary outcomes of this study were uptake of TB and DM testing.

Results: A total of 450,385 individuals were screened, of whom 18,109 had presumptive DM and 90,137 had presumptive TB. 14,550 of these individuals were presumptive for both DM and TB. The uptake of DM testing among those with presumptive diabetes was $26.1 \%$ while the uptake of TB testing among presumptive TB cases was $5.9 \%$. Despite efforts to promote bi-directional screening of TB and DM, the uptake of TB testing among pre-diabetes and diabetes cases was only $4.7 \%$, while the uptake of DM testing among MTB positive cases was $21.8 \%$.

Conclusion: While a high yield for TB was identified among pre-diabetics and diabetics along with a high yield of DM among individuals diagnosed with TB, there was a low uptake of TB testing amongst presumptive TB patients who were recorded as pre-diabetic or diabetic. Bi-directional screening for TB and DM which includes the integration of TB diagnostics, DM screening and TB-DM treatment within existing health care programs will need to address the operational challenges identified before implementing this as a strategy in public health programs.
\end{abstract}

Keywords: Bi-directional screening, Tuberculosis, Diabetes, Active-case-finding, Operational constraints

\footnotetext{
* Correspondence: mashal.basir@gmail.com

'Interactive Research \& Development, 4th Floor, Woodcraft Building, Plot No.

3 \& 3-A, Sector 47, Korangi Creek Road, Karachi, Pakistan

Full list of author information is available at the end of the article
}

(c) The Author(s). 2019 Open Access This article is distributed under the terms of the Creative Commons Attribution 4.0 International License (http://creativecommons.org/licenses/by/4.0/), which permits unrestricted use, distribution, and reproduction in any medium, provided you give appropriate credit to the original author(s) and the source, provide a link to the Creative Commons license, and indicate if changes were made. The Creative Commons Public Domain Dedication waiver (http://creativecommons.org/publicdomain/zero/1.0/) applies to the data made available in this article, unless otherwise stated. 


\section{Background}

Nearly one third of the world's population is infected with Mycobacterium tuberculosis (Mtb) [1]. A growing body of literature has established the association between tuberculosis (TB) and diabetes mellitus (DM) in various settings [2]. DM increases the overall risk of developing TB and contributes to adverse TB treatment outcomes such as delayed sputum conversion, treatment failure and relapse and death [2]. The global burden of diabetes is on the rise with the total number of people living with DM estimated to reach 438 million by 2030 [3]. The convergence of these two epidemics poses a significant challenge for health systems in low and middle-income countries. Studies in India and China found significant evidence for comorbidity at various health care levels including tertiary care facilities and primary healthcare clinics $[4,5]$. These studies led to important policy formulations that have mandated screening for $\mathrm{DM}$ among all $\mathrm{TB}$ patients and documentation of DM indicators within registries of the TB control program in these countries.

Screening, early-case detection and prompt treatment initiation is of relevance for both diseases. In 2009, the World Health Organization (WHO) and the International Union Against Tuberculosis and Lung Disease (IUATLD) recommended bi-directional TB-DM screening and integrated management for both diseases in high burden countries [6]. However, in low and middle-income countries, health systems are often weak and highly fragmented, including large and often poorly regulated private-sectors, and are not equipped to respond effectively to the dual burden of communicable and non-communicable diseases [7].

Pakistan is currently ranked fifth among high TB burden countries [1]. Nearly half of all cases remain undiagnosed and improving case-detection is an important target for the National TB Control Program (NTP) [1]. Pakistan also faces a rising burden of non-communicable diseases (NCDs), particularly Type II DM. It has the seventh highest country-burden for DM with an estimated prevalence of $6.8 \%$ in the adult population $[8,9]$. Similar to TB patients, diabetics are frequently under-diagnosed and an estimated 3.5 million adults in Pakistan are thought to be living with undiagnosed DM [9]. In Pakistan, TB control activities are under the purview of the National Tuberculosis Program (NTP), but routine screening for NCDs including DM is not currently recommended. Interventions also need to consider the private-sector that provides healthcare services to nearly three-quarters of all patients in the country [10].

In recent years, novel case-finding approaches for TB in the private-sector have been developed to enhance case-notifications through community-based screening, cash-incentives for referrals and linking case-notifications to the NTP disease surveillance system [11, 12]. Similar public-private models with a focus on integrated care can lower costs and improve health outcomes by shifting diseases-specific interventions to holistic, patient-centric approaches [13]. We investigated the feasibility of a bidirectional $\mathrm{TB}$ and $\mathrm{DM}$ screening program in the private health sector in Karachi, Pakistan as part of an enhanced case-finding intervention for TB through a social business approach (described below). The initiative builds upon previous interventions carried out in the area that were limited to targeting community-based screening for TB.

\section{Methods \\ Study design and setting}

A cross-sectional study of a bi-directional TB-DM screening program was conducted within a larger study investigating enhanced case-finding intervention for TB in the towns of Korangi, Landhi, Orangi, North Karachi and Nazimabad in Karachi, Pakistan, between February to November 2014. Community health workers (CHW) screened patients attending 80 general primary care, health provider clinics for TB and DM. The screening was based on a convenience consecutive sample. These clinics were primarily privatelyowned facilities of individual physicians and a small number of physician private group-practices, serving 50 or more patients on average per day.

\section{Screening and testing procedures}

CHWs were trained on TB screening and treatment according to NTP guidelines, and on DM screening according to the International Diabetes Federation (IDF) [14]. CHWs were subsequently assigned to private health providers' clinics where they verbally screened attendees for presumptive TB and DM. CHWs and health care providers were incentivized for referrals and case-detection. Presumptive TB was defined as cough of any duration, fever, unexplained weight-loss, night-sweats or hemoptysis. Patients with presumptive DM were defined as those aged over 30 years and with a family history of DM.

TB testing was carried out at purpose-built diagnostic centers called Sehatmand Zindagi (Healthy Life) located in Korangi and Nazimabad. The centers operate as a social business that aim to provide sustainable TB care in the private-sector. Revenue is generated through direct payment for tests by patients, including chest X-rays (USD 35 per test), spirometry (USD $2.5-5$ per test) and random blood glucose testing (USD 0.3-0.5 per test). Xpert MTB/ RIF testing was provided free-of-cost to all patients following a chest X-ray. The centers served as the site of treatment initiation and case-registration to the Provincial TB Program (PTP). The revenue generated is channeled into subsidizing TB diagnosis and treatment.

Individuals with presumptive TB were referred by the private health provider to the social business TB centers for a paid chest X-ray, followed by a free Xpert MTB/ RIF test. Patients with bacteriologically positive TB were 
tested for DM through a glucometer-based random blood sugar test (RBS) at the diagnostic centers. Individuals who came to the Centers with a previous diagnosis of TB or DM were re-tested using Sehatmand Zindagi Centers' Chest X-ray, Xpert MTB/RIF and glucometerbased random blood sugar tests to ensure reliable baseline measures for diagnosis rather than depending on external reports. 1180 individuals with known diabetes (Table 1), defined as all those who self-reported to be taking oral hypoglycemic medication, underwent glucometer based random blood sugar testing and then were channeled into the presumptive DM-TB arm (Fig. 1). Baseline data for previously diagnosed and newly diagnosed diabetes cases is indicated in Table 1. Previously diagnosed cases of diabetes were further classified as having controlled or uncontrolled diabetes. For the purpose of this analysis, uncontrolled diabetes was defined as RBS of greater than $200 \mathrm{mg} / \mathrm{dl}$ on glucometer testing. Those with presumptive DM were offered a paid (US \$2), point-of-care capillary blood-glucose test that was conducted by CHWs at the clinics. Cutoffs recommended by the International Diabetes Federation for resource-limited settings were used to categorize the patients as having DM $(\geq 200 \mathrm{mg} / \mathrm{dL}(11.1 \mathrm{mmol} / \mathrm{L}))$, Pre-DM (140-199 mg/ $\mathrm{dL}(7.8-11.0 \mathrm{mmol} / \mathrm{L}))$ or no diabetes $(<140 \mathrm{mg} / \mathrm{dL}(7.8$ $\mathrm{mmol} / \mathrm{L})$ ). Individuals with both presumptive TB and DM, were assigned to another diagnostic arm where the private health provider referred patients for both tests: a point of care glucometer test for DM and Chest X-ray and Xpert MTB/RIF testing at the diagnostic centers.

Data management and analysis

Data on DM and TB verbal screening results, referrals, and DM test results was recorded using a custom-built

Table 1 Baseline characteristics of patients identified as pre-diabetics and diabetics through community-based screening

\begin{tabular}{|c|c|c|c|c|c|c|c|c|c|c|}
\hline \multirow[t]{3}{*}{ Characteristics } & \multirow{2}{*}{\multicolumn{2}{|c|}{$\begin{array}{l}\text { Cases diagnosed } \\
\text { with Pre-diabetes }\end{array}$}} & \multirow{2}{*}{\multicolumn{2}{|c|}{$\begin{array}{l}\text { Newly diagnosed } \\
\text { cases of Diabetes }\end{array}$}} & \multicolumn{4}{|c|}{ Previously known cases of Diabetes } & \multirow{2}{*}{\multicolumn{2}{|c|}{ Total }} \\
\hline & & & & & \multicolumn{2}{|c|}{ Uncontrolled } & \multicolumn{2}{|c|}{ Controlled } & & \\
\hline & $n$ & $\%$ & $n$ & $\%$ & $n$ & $\%$ & $n$ & $\%$ & $n$ & $\%$ \\
\hline Total Number of patients & 726 & 100 & 547 & 100 & 901 & 100 & 279 & 100 & 2453 & 100 \\
\hline \multicolumn{11}{|l|}{ Sex } \\
\hline Male & 334 & 46 & 236 & 43.1 & 359 & 39.8 & 100 & 35.8 & 1029 & 41.9 \\
\hline Female & 392 & 54 & 311 & 56.9 & 542 & 60.2 & 179 & 64.2 & 1424 & 58.1 \\
\hline \multicolumn{11}{|l|}{ Age } \\
\hline$<30$ & 74 & 10.2 & 29 & 5.3 & 28 & 3.1 & 28 & 10 & 159 & 6.5 \\
\hline $30-39$ & 195 & 26.9 & 98 & 17.9 & 129 & 14.3 & 51 & 18.3 & 473 & 19.2 \\
\hline $40-49$ & 221 & 30.4 & 176 & 32.2 & 284 & 31.5 & 58 & 20.8 & 739 & 30.2 \\
\hline $50-59$ & 135 & 18.6 & 151 & 27.6 & 284 & 31.5 & 87 & 31.2 & 657 & 26.8 \\
\hline $60-69$ & 72 & 9.9 & 68 & 12.4 & 140 & 15.6 & 32 & 11.5 & 312 & 12.8 \\
\hline$>70$ & 29 & 4.0 & 25 & 4.6 & 36 & 4.0 & 23 & 8.2 & 113 & 4.5 \\
\hline \multicolumn{11}{|l|}{ Family History of Diabetes } \\
\hline Yes & 265 & 36.5 & 199 & 36.4 & 402 & 44.6 & 107 & 38.4 & 994 & 40.5 \\
\hline No & 461 & 63.5 & 348 & 63.6 & 499 & 55.4 & 172 & 61.6 & 1459 & 59.5 \\
\hline \multicolumn{11}{|c|}{ Current or Previous Smoking History } \\
\hline Yes & 88 & 12.1 & 55 & 10.1 & 32 & 3.6 & 37 & 13.3 & 212 & 8.6 \\
\hline No & 638 & 87.9 & 492 & 89.9 & 869 & 96.4 & 242 & 86.7 & 2241 & 91.4 \\
\hline \multicolumn{11}{|l|}{ Ethnicity } \\
\hline Sindhi & 29 & 4.0 & 9 & 1.6 & 15 & 1.7 & 9 & 3.2 & 62 & 2.5 \\
\hline Balochi & 09 & 1.2 & 5 & 0.9 & 8 & 0.9 & 4 & 1.4 & 26 & 1.1 \\
\hline Punjabi & 21 & 2.9 & 18 & 3.3 & 25 & 2.8 & 20 & 7.2 & 84 & 3.4 \\
\hline Pathan & 127 & 17.5 & 89 & 16.3 & 96 & 10.7 & 111 & 39.8 & 423 & 17.2 \\
\hline Muhajir & 518 & 71.3 & 367 & 67.1 & 734 & 81.5 & 110 & 39.4 & 1729 & 70.5 \\
\hline Other & 22 & 3.0 & 59 & 10.8 & 23 & 2.6 & 25 & 9.0 & 129 & 5.3 \\
\hline \multicolumn{11}{|l|}{ History of Hypertension } \\
\hline Yes & 210 & 28.9 & 75 & 13.7 & 273 & 30.3 & 171 & 61.3 & 728 & 29.7 \\
\hline No & 516 & 71.1 & 472 & 86.3 & 628 & 69.7 & 109 & 38.7 & 1725 & 70.3 \\
\hline
\end{tabular}




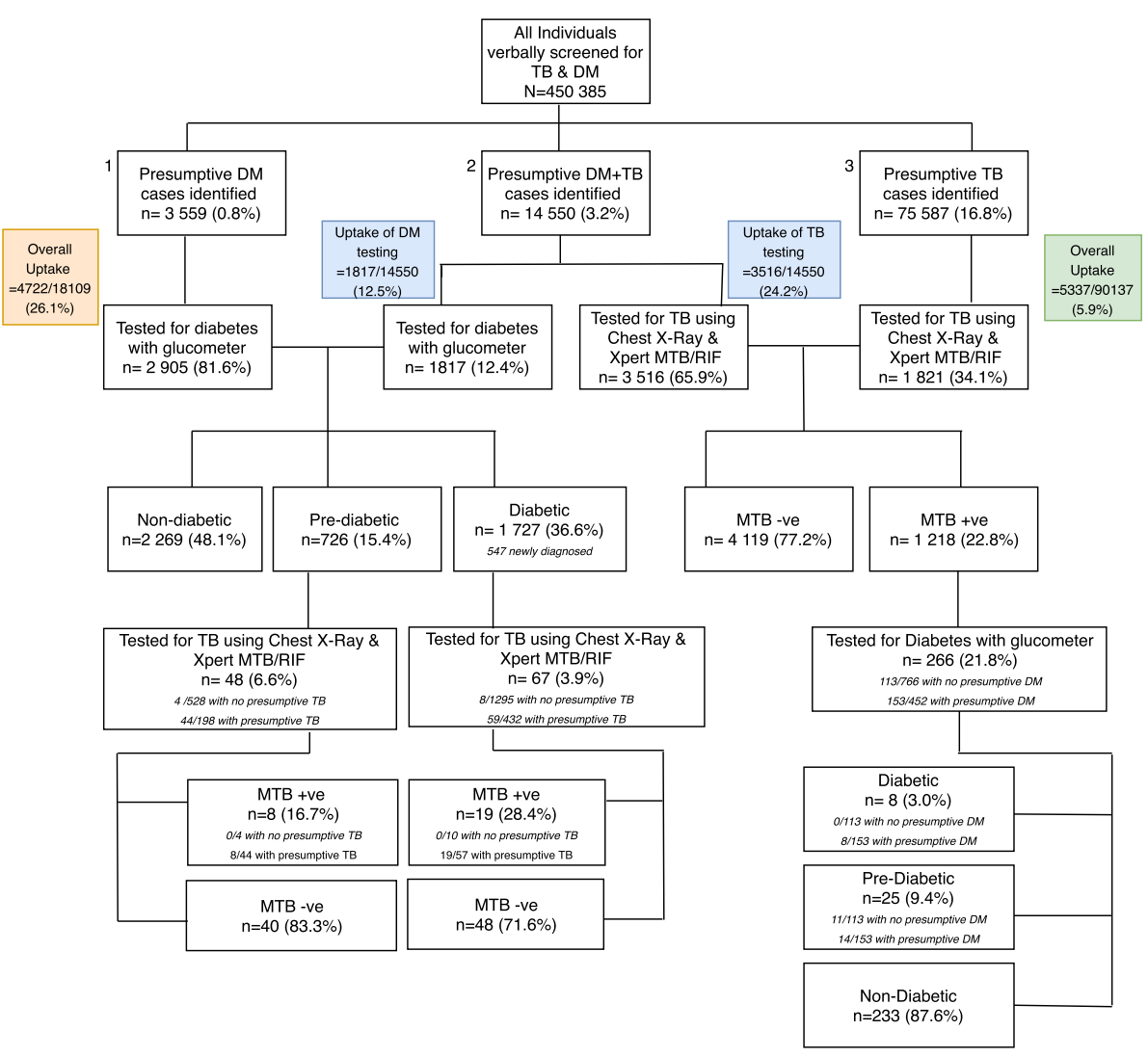

KEY

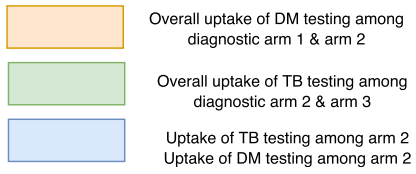

Fig. 1 Diagnostic algorithm and results of TB-DM bidirectional screening Feb-Nov, 2014, Karachi, Pakistan

mobile-phone application. Other variables ascertained at baseline included family-history of diabetes, smoking history, ethnicity, and past history of TB treatment (Table 2). Patients' chest X-ray and Xpert MTB/RIF results at the diagnostic centers were recorded using OpenMRS, an Open source enterprise electronic medical record system platform. The mobile and web applications included several data validation checks to ensure data-accuracy. Field supervisors and project management staff were responsible for overall data-validation and accuracy including reporting to the NTP.

Frequency analysis of baseline demographic characteristics of patients diagnosed with DM and Pre-DM/Impaired Fasting and TB were performed. The proportions of individuals diagnosed with Pre-DM and DM that had TB symptoms and subsequently underwent Xpert MTB/ RIF testing and diagnosed with TB were calculated. The proportion of newly diagnosed DM cases among $\mathrm{TB}$ patients was calculated. Differences between the DM and Pre-DM groups were assessed for statistical significance using chi-square or Pearson's test (Table 2). All statistical analysis was carried out using Stata 12.1 (College Station, TX: StataCorp LP).

The primary study outcomes were uptake of TB testing and uptake of DM testing. Overall uptake of TB testing, overall uptake of DM testing, uptake of TB testing and uptake of DM testing among patients presumptive for both DM \& TB were computed (Fig. 1). Additionally, uptake of TB testing among pre-diabetic and diabetic patients was assessed by comparing the proportion of pre-diabetic and diabetic individuals that underwent Chest X-ray and Xpert MTB/RIF testing to the proportion within each arm who were presumptive for TB. Uptake of DM testing among MTB positive patients was computed by comparing the proportion of MTB positive patients who underwent a glucometer-based random 
Table 2 Baseline characteristics and results of glucometer testing conducted on TB patients through community-based TB-DM screening

\begin{tabular}{|c|c|c|c|c|c|c|c|}
\hline & \multicolumn{2}{|c|}{ Persons with TB Tested for DM* } & \multicolumn{2}{|c|}{ Pre-Diabetes* } & \multicolumn{2}{|c|}{ Newly Diagnosed DM cases } & \multirow[t]{2}{*}{$p$-valuet } \\
\hline & $n$ & $\%$ & $n$ & $\%$ & $n$ & $\%$ & \\
\hline Total & 266 & 100 & 25 & 100 & 8 & 100 & \\
\hline \multicolumn{8}{|l|}{ Sex } \\
\hline Male & 108 & 40.6 & 13 & 48.0 & 4 & 50.0 & \multirow[t]{2}{*}{0.99} \\
\hline Female & 158 & 59.4 & 12 & 48.0 & 4 & 50.0 & \\
\hline \multicolumn{8}{|l|}{ Age (years) } \\
\hline$<20$ & 60 & 22.6 & 4 & 16.0 & 0 & 0.0 & \multirow[t]{4}{*}{$<0.01$} \\
\hline $20-29$ & 93 & 35.0 & 5 & 20.0 & 0 & 0.0 & \\
\hline $30-39$ & 43 & 16.2 & 2 & 8.0 & 1 & 12.5 & \\
\hline 40 and above & 67 & 25.2 & 14 & 56.0 & 7 & 87.5 & \\
\hline \multicolumn{8}{|l|}{ Family History of $\mathrm{DM}^{*}$} \\
\hline Yes & 32 & 12.0 & 1 & 4.0 & 3 & 37.5 & \multirow[t]{2}{*}{0.97} \\
\hline No & 234 & 88.0 & 24 & 96.0 & 5 & 62.5 & \\
\hline \multicolumn{8}{|l|}{ Current or Previous Smoking History } \\
\hline Yes & 21 & 7.9 & 7 & 28.0 & 2 & 25.0 & \multirow[t]{2}{*}{$<0.01$} \\
\hline No & 245 & 92.1 & 18 & 72.0 & 6 & 75.0 & \\
\hline \multicolumn{8}{|l|}{ Ethnicity } \\
\hline Sindhi & 14 & 5.3 & 2 & 8.0 & 2 & 25.0 & \multirow[t]{6}{*}{0.11} \\
\hline Balochi & 2 & 0.8 & 0 & 0.0 & 0 & 0.0 & \\
\hline Punjabi & 8 & 3.0 & 1 & 4.0 & 0 & 0.0 & \\
\hline Pathan & 118 & 44.4 & 5 & 20.0 & 3 & 37.5 & \\
\hline Muhajir & 91 & 34.2 & 12 & 48.0 & 1 & 12.5 & \\
\hline Other & 33 & 12.4 & 3 & 12.0 & 1 & 12.5 & \\
\hline \multicolumn{8}{|l|}{ MTB Burden } \\
\hline Negative (clinically diagnosed) & 28 & 10.5 & 3 & 12.0 & 2 & 25.0 & \multirow[t]{4}{*}{0.85} \\
\hline Very low-low & 90 & 33.8 & 9 & 34.0 & 2 & 25.0 & \\
\hline Medium & 90 & 33.8 & 9 & 36.0 & 3 & 37.5 & \\
\hline High & 58 & 21.8 & 4 & 16.0 & 1 & 12.5 & \\
\hline \multicolumn{8}{|l|}{ Type of TB } \\
\hline New & 229 & 86.1 & 22 & 88.0 & 7 & 87.5 & \multirow[t]{2}{*}{0.75} \\
\hline Retreatment & 37 & 13.9 & 3 & 12.0 & 1 & 12.5 & \\
\hline
\end{tabular}

"The cut-offs for RBS testing were taken from the International Diabetes federation

† The $\mathrm{p}$-value denotes the level of significance found when testing differences in sociodemographic characteristics between individuals identified as new DM cases and those identified as pre-DM, among all TB patients who had a glucometer blood sugar test

blood sugar test to the proportion within that arm who were presumptive for DM. The yield of TB among individuals identified with Pre-DM and DM was computed as the proportion of individuals who tested positive for $\mathrm{TB}$ among all individuals with $\mathrm{DM}$ and pre-DM who underwent TB testing via chest X-ray and Xpert MTB/ RIF in this project. The yield of DM among individuals diagnosed with TB was calculated as the proportion of individuals identified with $\mathrm{DM}$ and Pre-DM among all individuals with TB who underwent glucometer-based RBS testing in this project.

\section{Results}

A total of 450,385 individuals were verbally screened during the study period. Of those verbally screened, $3559(0.8 \%)$ were identified as having presumptive DM, $14,550(3.2 \%)$ with both presumptive $\mathrm{DM}$ and $\mathrm{TB}$, and $75,587(16.8 \%)$ as having presumptive TB only (Fig. 1). The overall uptake of DM testing among those identified with presumptive diabetes was $26.1 \%$ while the overall uptake of TB testing among those with presumptive TB was $5.9 \%$ (Fig. 1). Individuals with a presumptive diagnosis of both DM and TB were referred for both a 
glucometer based random blood sugar test and a Chest X-ray and Xpert MTB/RIF test. Among the group that was presumptive for both $\mathrm{DM}$ and $\mathrm{TB}$, the uptake of $\mathrm{DM}$ testing was $12.5 \%$ and the uptake of $\mathrm{TB}$ testing was 24.2\% (Fig. 1).

\section{Baseline socio-demographic and clinical characteristics of pre-diabetic and diabetic patients}

The majority of pre-diabetic and diabetic patients self-identified as Muhajir (the most common ethnic group in Karachi). Of those patients who tested positive for diabetes, 547 (31.6\%) were newly diagnosed. Of the 1180 previously known diabetes cases, 901 (76.4\%) had blood sugar levels above the recommended range for optimal diabetics control (Table 1). A higher proportion of females were identified with pre-diabetes and diabetes (58.1\%) and a family history of diabetes, history of smoking and history of hypertension was reported in 40.5, 8.6 and $29.7 \%$ of diabetics respectively. A history of hypertension was reported in a higher proportion of previously known diabetics with controlled blood sugar levels (61.3\%) compared to known diabetics with poorly controlled blood sugar levels (30.3\%), newly diagnosed diabetics $(13.7 \%)$ and pre-diabetics (28.9\%) (Table 1$)$.

\section{Baseline socio-demographic characteristics of TB patients} The yield of diabetes among TB patients was found to be significantly higher in patients $\geq 40$ years of age $(p$-value <0.01) and among smokers compared to non-smokers (p-value <0.01) (Table 2). Gender, MTB burden, ethnicity and past history of TB treatment were not associated with an increased yield of DM (Table 2).

\section{Uptake of diabetes testing}

A total of $4722(26.1 \%)$ individuals with presumptive DM underwent glucometer testing, with 726 (15.4\%) identified as pre-diabetic (198 of whom had both presumptive TB and DM), and 1727 (36.6\%) diagnosed with diabetes (432 of whom had both presumptive DM and TB). Among those diagnosed with TB, a higher proportion of those with presumptive DM consented for DM screening using a glucometer, than those without presumptive DM $(33.8 \%$ vs $14.7 \%, p<0.001)$. Among those who underwent glucometer testing, 25 (9.4\%) were identified as pre-diabetics and $8(3 \%)$ as diabetics. Of those diagnosed with diabetes and pre-diabetes, 100 and 56\% $(14 / 25)$ had presumptively screened positive for DM (Fig. 1). The median age of individuals identified as presumptive for TB and DM was 34 years (IQR 25-45), and 47 years (IQR $38-55$ ) respectively. The proportion of females among those identified as presumptive for TB and DM, was 47.4 and $50.1 \%$ respectively.

\section{Uptake of TB testing}

A high proportion $(22.8 \%)$ of individuals with presumptive TB, tested positive for MTB on Xpert MTB/RIF. Overall, the uptake of screening for tuberculosis was quite low among prediabetics and diabetics. 6.6\% of prediabetic cases and $3.9 \%$ of diabetic cases underwent TB testing via Chest X-ray and Xpert MTB/RIF. However, the uptake was significantly higher among those who had presumptive TB $(22.2 \%$ vs $0.8 \%$ among pre-diabetics; and $13.7 \%$ vs $0.62 \%$ among diabetics). Of the 153 who consented for TB testing, 153 had a chest X-ray and among these 115 (75.2\%) underwent Xpert MTB/RIF testing (the remainder were unable to expectorate sputum and excluded from further diagnostic evaluation) (Table 3). A total of 8 pre-diabetics (16.7\%) and 19 diabetics (28.4\%) tested positive for TB on Xpert MTB/RIF testing (Fig. 1).

\section{Discussion}

Our study evaluated bi-directional TB-DM screening in a high TB and DM prevalence setting. In this study, the yield of $\mathrm{TB}$ among those with diabetes and pre-diabetes was $23.5 \%$ while the yield of pre-DM/DM among individuals who had tested positive for TB was $12.4 \%$ (Fig. 1). However, it is important to note that the yield of $\mathrm{TB}$ in our sample has a great risk of selection bias. Notably all had presumptive TB; the number of diabetics without presumptive $\mathrm{TB}$ who took up screening was too small to comment on the yield of TB among those tested for TB without any suggestive symptoms. A number of studies, as well as the Pakistan TB prevalence survey have identified a large proportion of TB cases with only Chest $\mathrm{X}$-ray abnormalities and no TB symptoms supporting its use as the front-line screening procedure [15]. Therefore, we aimed to test all individuals for TB using chest radiography, regardless of symptoms. In circumstances where chest X-rays are unavailable symptomatic screening can be used as an alternative. In this study, a high yield of TB was recorded among those with TB symptoms. We were unable to assess the effectiveness of the chest X-ray in identifying additional cases of TB as the majority of cases referred were also symptomatic for TB.

While our study supports the growing body of literature highlighting the association between TB and DM [8] it primarily identifies a number of operational constraints that programs may need to address during larger scale TB-DM screening interventions. A high yield of $\mathrm{TB}$ was found among the diabetics and pre-diabetics within our study population. The uptake of diagnostic evaluation for TB was low, and mainly confined to those who had symptoms suggestive of TB. In addition, since physician prescriptive practices also significantly affect patient behavior, it is possible that only diabetics with clinical suspicion of TB were actively referred [16]. Additional constraints in testing diabetics for TB included 
Table 3 Xpert MTB/RIF test results of individuals diagnosed with Diabetes and Pre-Diabetes through community-based screening

\begin{tabular}{|c|c|c|c|c|c|c|}
\hline & \multicolumn{2}{|c|}{ Individuals identified with Pre-Diabetes } & \multicolumn{2}{|c|}{ Individuals identified with Diabetes Mellitus } & \multicolumn{2}{|l|}{ Total } \\
\hline & Number(n) & Percent (\%) & Number $(n)$ & Percent (\%) & Number $(n)$ & Percent (\%) \\
\hline Total & 726 & 100 & 1727 & 100 & 2453 & 100 \\
\hline Underwent Paid Chest X-Ray & 62 & 8.5 & 91 & 5.3 & 153 & 6.2 \\
\hline Underwent Xpert MTB/RIF & 48 & 77.4 & 67 & 73.6 & 115 & 75.2 \\
\hline Diagnosed with TB & 8 & 16.7 & 19 & 28.4 & 27 & 23.5 \\
\hline Started on treatment for TB & 8 & 100 & 19 & 100 & 27 & 100 \\
\hline
\end{tabular}

the price for the chest X-ray and the distance to the centers for diagnostic evaluation. Our experience suggests that in a pay for testing model only diabetics with a high suspicion of TB will be referred, as both providers and patients perceived the costs and time associated with the $\mathrm{X}$-ray as key barriers. Also, approximately a quarter of patients who agreed to undergo a chest X-ray, could not be tested on Xpert MTB/RIF due to their inability to expectorate sputum and this further limited the number of diabetics among whom the yield of TB could be assessed.

Our early experience of implementing TB-DM screening in a programmatic setting in the private-sector offers a number of lessons for potential interventions that can be carried out at scale. Firstly, physicians must be trained to refer diabetics for routine screening for TB, as is carried out for other diabetes-associated complications such as nephropathy or retinopathy. Patients also need to be appropriately counseled regarding the risks of developing $\mathrm{TB}$, particularly those with poorly controlled diabetes since they are at higher risk for developing TB [17]. Secondly, user-fees for the X-ray and distance to the TB centers limited the number of diabetics undergoing TB screening in our program. It can be assumed that user fee is likely a barrier to uptake of preventive health services such as screening for TB and DM. As a preventative intervention with potential public health benefits, cost-free or subsidized services may increase uptake of TB testing. This may likely encourage pre-diabetics and diabetics to undergo screening, particularly those who have no symptoms of TB, and will help decrease the case-detection gap for TB. Public health practitioners and program managers that are eager to roll out a bi-directional TB-DM screening program in the private sector, should incorporate greater cost subsidies for patients in the program budget. Third, scale-up of TB screening in diabetics will require ensuring adequate sputum expectoration from diabetics through use of nebulizers and appropriate counseling. Commercial labs in the private-sector and most healthcare providers do not routinely carry out such procedures and will require capacity-strengthening. Existing specialized diabetes treatment centers with high volumes of known diabetics or private-practices of diabetes and endocrine specialists can be targeted to refer diabetics for TB screening.
Over $50 \%$ of diabetics and pre-diabetics identified in our program were newly diagnosed further highlighting the burden of undiagnosed diabetes and the need to introduce affordable and accessible diabetes screening in the private-sector at scale as a prelude for enhanced case-detection for TB.

Screening for DM among TB patients presented fewer operational challenges in our model as patients were offered screening during the time of registration for $\mathrm{TB}$ treatment. The yield of pre-DM and DM identified among individuals with TB in our program was higher (12.4\%) than the DM prevalence in the general population of Pakistan (6.9\%) [8]. This is consistent with findings from similar studies from India that have reported a prevalence between 5 and $54.1 \%$ of DM in TB patients [18-20]. Routine screening for DM among TB patients should therefore be considered at TB management facilities in Pakistan.

Diabetes screening for TB patients can be best carried out at the time of initiation of TB treatment and registration. Low-cost, point-of-care tests for diabetes are recommended as patients are more likely to consent to them although their results are less accurate. Interventions will need to ensure that these tests are available either at laboratories or at provider clinics where TB treatment is initiated for private-sector patients and data on diabetes screening and control is captured as part of the TB surveillance system. Integrated delivery units such as the ones developed by our project offer a potential solution where TB diagnostics, DM screening and TB-DM treatment can be offered in one facility with supporting systems for data capture and monitoring of outcomes.

\section{Conclusion}

This study provides evidence for the operationalization and the associated challenges of bi-directional screening of TB and DM in the private health sector of low-middle income countries. A high yield for TB was identified among diabetics and pre-diabetics in our study population, however the overall uptake of bi-directional screening remained low. A large number of diabetics were newly identified suggesting the need for implementation of collateral active case-finding initiatives for DM. This study also provides a potential model for integration of $\mathrm{TB}$ 
diagnostics, DM screening and TB-DM treatment within one facility that can be similarly adapted for other areas of public health. The private sector can be engaged cost-effectively through trainings and incentives to help achieve increased case-detection for both TB and DM.

\section{Abbreviations}

DM: Diabetes Mellitus; FBS: Fasting Blood Sugar; IDF: International Diabetes Federation; MTB: Mycobacterium tuberculosis; NCD: Non-Communicable Disease; NNT: Numbers needed to test; NTP: National Tuberculosis Program; Pre-DM: Pre-Diabetes Mellitus; PTP: Provincial Tuberculosis Program; RBS: Random Blood Sugar; TB: Tuberculosis; WHO: World health Organization

\section{Acknowledgements}

The authors would like to thank Stop TB Partnership for supporting the interventions described in this paper, and Dr. Amyn A. Malik for providing invaluable feedback.

\section{Funding}

The authors did not receive any specific grant for research from funding agencies in the public, commercial, or not-for-profit sectors.

\section{Availability of data and materials}

The datasets used and analyzed during the current study are available from the corresponding author on reasonable request.

\section{Author's contributions}

MSB, SMAZ, and SK were involved in conception of the study, finalizing the study design, supervising field activities and conducting data collection. MSB drafted the manuscript, formatted the tables, figures and references, and has brought the manuscript to its current form. MSB, SSH, and SMAZ conducted the literature review, data analysis and data interpretation. SK provided crucial technical knowledge about $\mathrm{TB}$. $\mathrm{HH}$ reviewed the manuscript to ensure clinical aspects of the bidirectional TB-DM screening program were in accordance with international guidelines. RF was instrumental in designing the screening algorithms for the bi-directional TB-DM program. SK, HH, RF and AJK critically reviewed the manuscript for intellectual content and finalized the manuscript. All authors read and approved the final manuscript.

\section{Author's information}

MSB was responsible for initiating the diabetes pilot for community-based screening, diagnosis and treatment at IRD. She is currently pursuing her Master of Public Health degree at Columbia University. SSH is a public health researcher who is involved with impact evaluations of new diagnostic technologies such as Xpert MTB/RIF testing and computer-aided detection for TB in the Pakistani population. Her on-going research focuses on impact of anti-tuberculosis treatment on $\mathrm{HbA1C}$ levels among Diabetics with TB. SMAZ is a medical epidemiologist and Program Manager on the TB-DM bidirectional-screening program. SK is a program development expert and was the Director of Strategy and Operations at IRD when the TB-DM bidirectional-screening program was rolled out. $\mathrm{HH}$ is a public health professional who serves as Country Director of IRD Bangladesh and was instrumental in scaling drug resistant TB (DR-TB) treatment sites to 10 cities across Pakistan through Global Fund support. RF is a Professor at London School of Hygiene and Tropical Medicine, and is Chief Scientist for a multi-country RCT for treatment of HIV-associated chronic lung disease. AJK is an epidemiologist and social entrepreneur, currently testing social business models for the delivery of affordable lung health and diabetes care in Bangladesh, Indonesia, and Pakistan. Aamir was the driving force behind launching the bidirectional TB-DM screening program in Karachi.

\section{Ethics approval and consent to participate}

The study was approved by the Institutional Review Board (IRB) at Interactive Research and Development (IRD), Karachi, Pakistan. The IRB is registered with the U.S. Department of Health and Human Services (DHHS), Office for Human Research Protections (IRB\#00005148). The ethics committee formally approved using verbal consent. Verbal consent was obtained from participants prior to conducting blood glucose and Xpert MTB/RIF tests and documented on the screening forms. De-identified data from the project was used for data analysis.

\section{Consent for publication}

Not applicable.

\section{Competing interests}

The authors declare that they have no competing interests.

\section{Publisher's Note}

Springer Nature remains neutral with regard to jurisdictional claims in published maps and institutional affiliations.

\section{Author details}

'Interactive Research \& Development, 4th Floor, Woodcraft Building, Plot No. 3 \& 3-A, Sector 47, Korangi Creek Road, Karachi, Pakistan. ${ }^{2}$ Community Health Solutions, 9th Floor, Al-Tijarah Building, Main Shahrah-e-Faisal, Karachi, Pakistan. ${ }^{3}$ Interactive Research \& Development, London School of Hygiene and Tropical Medicine, Keppel St, Bloomsbury, London WC1E 7HT, UK.

Received: 31 August 2018 Accepted: 26 February 2019

Published online: 06 March 2019

References

1. World Health Organization. World Health Organization Global Tuberculosis report 2015.

2. Baker MA, Harries AD, Jeon CY, Hart JE, Kapur A, Lönnroth $K$, Ottmani SE, Goonesekera SD, Murray MB. The impact of diabetes on tuberculosis treatment outcomes: a systematic review. BMC Med. 2011;9(1):1.

3. Sicree R, Shaw J, Zimmet P, Heart Bl. The global burden. Diabetes and impaired glucose tolerance Baker IDI Heart and Diabetes Institute. 2010.

4. Lönnroth K, Roglic G, Harries AD. Improving tuberculosis prevention and care through addressing the global diabetes epidemic: from evidence to policy and practice. The Lancet Diabetes \& Endocrinology. 2014;2(9):730-9.

5. Harries AD, Satyanarayana S, Kumar AM, Nagaraja SB, Isaakidis $P$, Malhotra $S$, Achanta S, Naik B, Wilson N, Zachariah R, Lönnroth K. Epidemiology and interaction of diabetes mellitus and tuberculosis and challenges for care: a review [review article]. Public Health Action 2013;3(Supplement 1):3-9.

6. World Health Organization, 2011. Collaborative framework for care and control of tuberculosis and diabetes. http://www.who.int/diabetes/ publications/tb_diabetes2011/en/. Accessed 5 July 2016.

7. Nigatu T. Integration of HIV and noncommunicable diseases in health care delivery in low-and middle-income countries. Prev Chronic Dis. 2012;9.

8. International Diabetes Federation, Diabetes Atlas, 7th edn. Brussels: International Diabetes Federation, 2015. http://www.diabetesatlas.org. Accessed 12 Aug 2016.

9. International Diabetes Federation. IDF global diabetes scorecard. Brussels: International Diabetes Federation, 2014. https://www.idf.org/our-activities/ advocacy-awareness/resources-and-tools/53-global-diabetes-scorecard.html. Accessed 6 June 2016

10. Federal Bureau of Statistics, Statistics Division. Pakistan Social and Living Standards Measurement Survey 2014-15. Islamabad: Government of Pakistan. http://www.pbs.gov.pk/sites/default/files//ps/m/publications/PSLM_ 2014-15_National-Provincial-District_report.pdf. Accessed 20 Aug 2016.

11. Khan AJ, Khowaja S, Khan FS, Qazi F, Lotia I, Habib A, Mohammed S, Khan $\mathrm{U}$, Amanullah F, Hussain H, Becerra MC. Engaging the private sector to increase tuberculosis case detection: an impact evaluation study. Lancet Infect Dis. 2012;12(8):608-16.

12. Creswell J, Khowaja S, Codlin A, Hashmi R, Rasheed E, Khan M, Durab I, Mergenthaler C, Hussain O, Khan F, Khan AJ. An evaluation of systematic tuberculosis screening at private facilities in Karachi, Pakistan. PLoS One. 2014;9(4):e93858.

13. The Global Fund. Universal Health coverage, 2018. https://www. theglobalfund.org/media/5913/publication_universalhealthcoverage_ focuson_en.pdf?u=636825488920000000. Accessed 17 Oct 2018.

14. International Diabetes Federation. Global guideline for Type 2 Diabetes. Brussels: International Diabetes Federation; 2015. https://www.idf.org/elibrary/guidelines/79-global-guideline-for-type-2-diabetes. Accessed 7 Feb 2016.

15. Qadeer E, Fatima R, Yaqoob A, Tahseen S, Haq MU, Ghafoor A, Asif M, Straetemans M, Tiemersma EW. Population based national tuberculosis prevalence survey among adults (> 15 years) in Pakistan, 2010-2011. PLoS One. 2016;11(2):e0148293. 
16. Gabay G. Perceived control over health, communication and patientphysician trust. Patient Educ Couns. 2015;98(12):1550-7.

17. Dooley KE, Chaisson RE. Tuberculosis and diabetes mellitus: convergence of two epidemics. Lancet Infect Dis. 2009;9(12):737-46. https://doi.org/10.1016/ S1473-3099(09)70282-8.

18. Viswanathan V, Kumpatla S, Aravindalochanan V, Rajan R, Chinnasamy C, Srinivasan R, Selvam JM, Kapur A. Prevalence of diabetes and pre-diabetes and associated risk factors among tuberculosis patients in India. PLoS One. 2012;7(7):e41367.

19. Balakrishnan S, Vijayan S, Nair S, Subramoniapillai J, Mrithyunjayan S, Wilson N, Satyanarayana S, Dewan PK, Kumar AM, Karthickeyan D, Willis M. High diabetes prevalence among tuberculosis cases in Kerala, India. PLoS One. 2012;7(10):e46502

20. Kumar A, Jain DC, Gupta D, Satyanarayana S, Zachariah R, Harries AD. Screening of patients with tuberculosis for diabetes mellitus in India. Tropical medicine \& international health: TM \& IH. 2013.

Ready to submit your research? Choose BMC and benefit from:

- fast, convenient online submission

- thorough peer review by experienced researchers in your field

- rapid publication on acceptance

- support for research data, including large and complex data types

- gold Open Access which fosters wider collaboration and increased citations

- maximum visibility for your research: over $100 \mathrm{M}$ website views per year

At BMC, research is always in progress.

Learn more biomedcentral.com/submissions 